\title{
一次太阳射电分米波 $\mathbf{U}$ 型爆发的观测特性 *
}

\author{
姚金兴 A. Tlamicha ${ }^{(1)}$ 魏奉思 ${ }^{(2)}$ 于兴凤 \\ (中国科学院紫金山天文台,南京 210008; (1) Astronomical Institute Ondrejov Observatory, 25165 Czech; \\ (2) 中国科学院空间科学与应用研究中心, 日球物理数值开放研究实验室, 北京 100080)
}

\section{关键词太阳耀斑 U 型爆 源参数}

太阳射电反转 U 型爆发, 因它在频谱仪上的观测形态象反转过来的 U 而得名. 它对研究 太阳耀斑的物质执射和粒子加速有重要意义. 迄今为止, $\mathrm{U}$ 型爆发绝大多数在低于 $0.5 \mathrm{GHz}$ 的频率上出现, 在 $1.1 \sim 1.7 \mathrm{GHz}$ 上只报道了一例 ${ }^{[1]}$. 本文中将介绍的在 $1.0 \sim 2.8 \mathrm{GHz}$ 上的 $\mathrm{U}$ 型爆 (见图 1 和图 2), 在国际上尚属首例. 这是由捷克 Ondrejov天文台观测的, 时间分辨率 可达 $1 \mathrm{~ms}$, 频率分辨率为 $10 \mathrm{MHz}$.

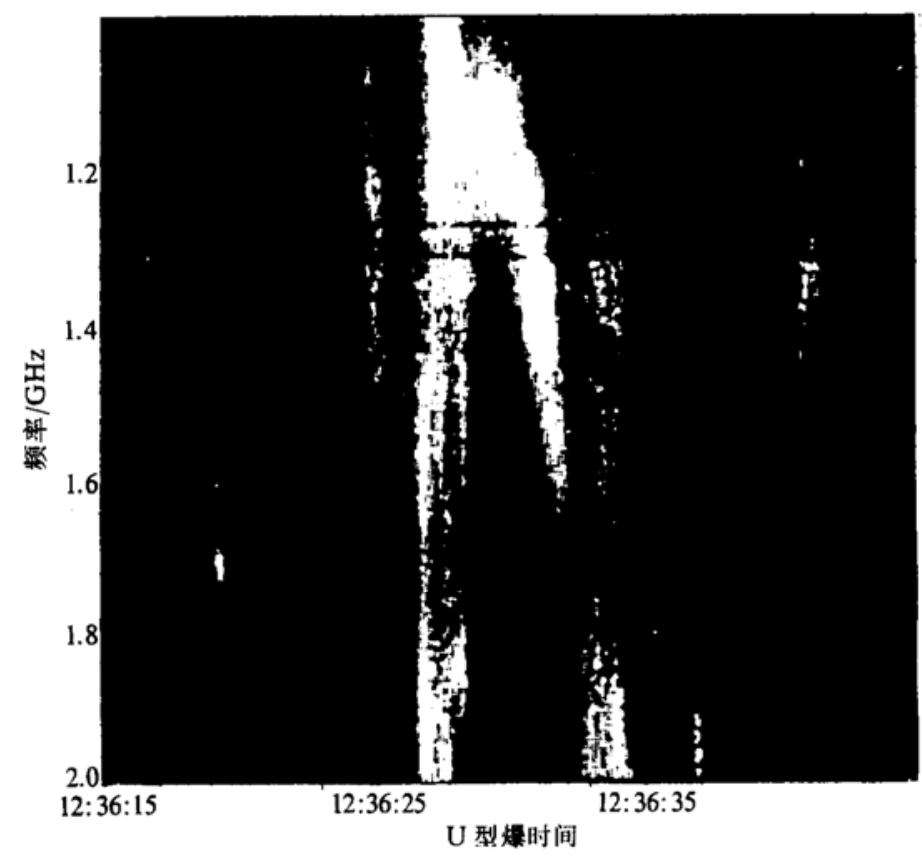

图 $1 \quad 1992-08-22 \quad 1.0 \sim 2.8 \mathrm{GHz}$ U 型爆的灰标图 由捷克 Ondrejov天文台观测到

1995-07-04 收稿, 1995-10-26 收修改稿

* 国家自然科学重大基金资助项目 


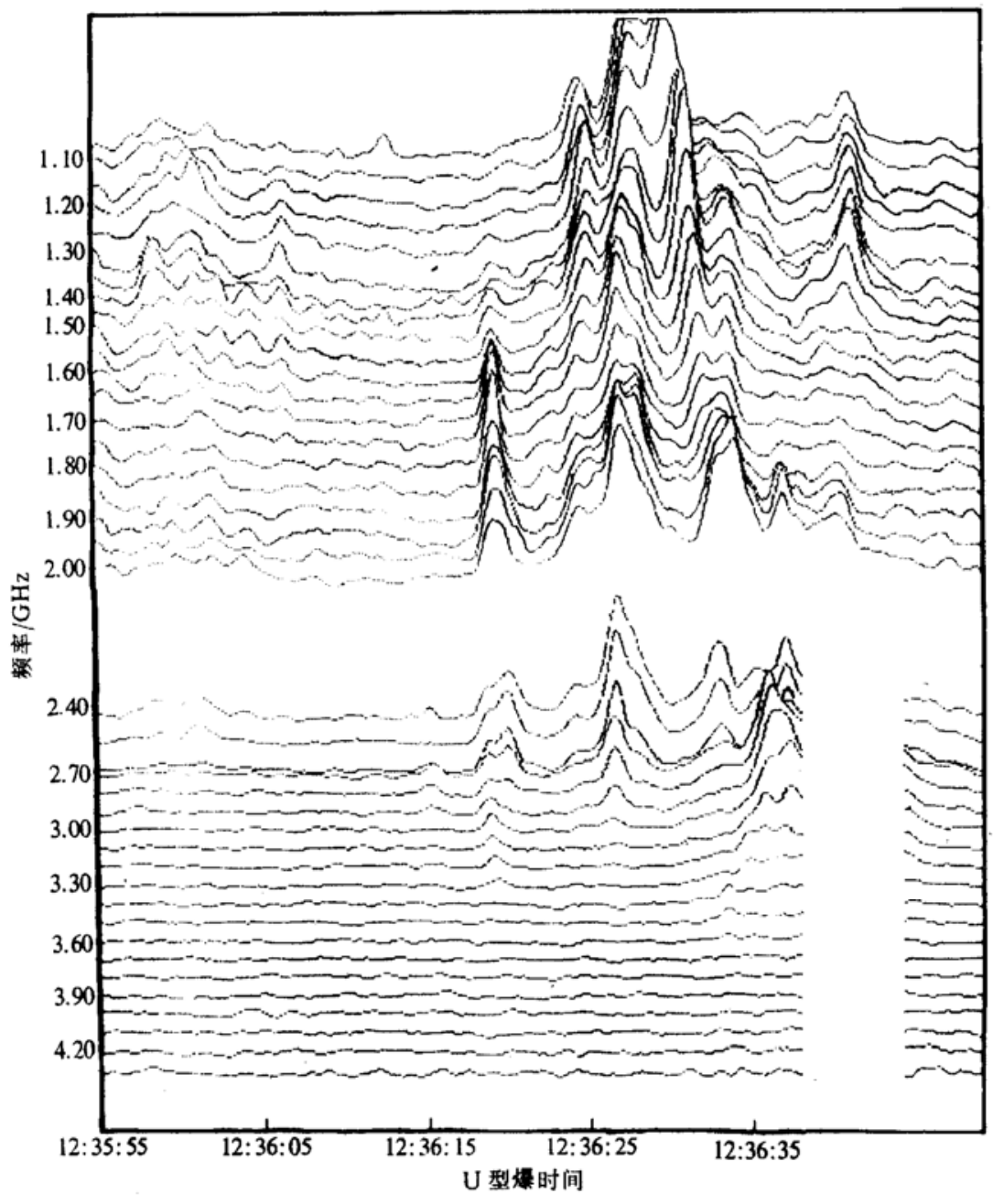

图 2 U 型爆的时间轮廊

\section{1 资料简介}

$\mathrm{U}$ 型爆发通常发生在日冕高层, 即相应于米波段的等离子体层. 这个 U 型爆的起始频率 为 $2.8 \mathrm{GHz}$, 这在日冕底层. 这个爆发的上升段频率为 $2.8 \sim 1 \mathrm{GHz}$, 下降段的为 $1.12 \sim$ $1.75 \mathrm{GHz}$, 转换频率 (turning frequency) 为 $1.12 \mathrm{GHz}$ (参见图 1). 上升段和下降段的频漂几乎 是不变的, 分别为 1.25 和 $0.225 \mathrm{GHz} / \mathrm{s}$. 上升段的最大带宽约为 $1.8 \mathrm{GHz}$, 而下降段的约为 $0.35 \mathrm{GHz}$. 它伴随有 $\mathrm{C} 1.2$ 级软 $\mathrm{X}$ 射线爆和 $\mathrm{H} \alpha$ 亚耀斑 ${ }^{[2]}$.

\section{2 源的物理参数}

目前大都认为, $U$ 型爆是由电子束流在封闭磁环中运动激发等离子体辐射形成的. 因此 可由爆发参数推知磁环中的物理状态. 由于非常快的频漂, 可假设上升段的环腿为沿径向的 圆柱体, 而下降段的环腿为与上升的成某一角 $\theta$ 的圆柱体. 


\section{1 电子束流的速度}

采用 Newkirk 的日冕电子分布模型 ${ }^{[3]}$ :

$$
N_{\mathrm{e}}=N_{o} 10^{4.32 R_{\odot} / R},
$$

其中 $R_{\odot}$ 为太阳半径, $R$ 为从太阳中心量起的距离. 取 $2.8 \mathrm{GHz}$ 的等离子体层在光球上 $5000 \mathrm{~km}$ 地方. 故对上升段, $2.8 \sim 1 \mathrm{GHz}$ 的频率范围相应于光球上空 $5000 \mathrm{~km}$ 到 190000 $\mathrm{km}$. 按照文献[4],有

$$
\Delta t=\left(\frac{1}{v}-\frac{\cos \alpha}{c}\right) \Delta R+\tau(\alpha),
$$

$\Delta t$ 为在 $2.8 \mathrm{GHz}$ 和 $1 \mathrm{GHz}$ 上达到极大的时刻差, $\Delta R$ 为两者的径向距离, $\tau(\alpha)$ 为射电波在折 射指数不为 1 介质中传播的一个改正系数. 按照文献 [4]的公式, 计算得 $\tau(\alpha) \approx 0.13 \mathrm{~s} . \alpha$ 为 日心经度. 这个爆发发生在 NOAA7260 活动区, $\alpha$ 为 $60^{\circ}[2]$. 从图 1 量得 $\Delta t \approx 1.4 \mathrm{~s}$. 从(2) 式,算得上升段的电子束流速度约为 $0.38 c$.

对下降段,类似于 (2)式有

$$
\Delta t=\left(\frac{1}{v}-\frac{|\cos (\alpha+\theta)|}{c}\right) \frac{\Delta R}{\cos \theta}+\tau(\alpha),
$$

其中 $\Delta t$ 为 1.12 和 $1.75 \mathrm{GHz}$ 达到极大的时间差, $\Delta t \approx 2.8 \mathrm{~s}, \Delta R$ 为两者的径向距离, $\Delta R \approx$ $85000 \mathrm{~km}, \tau(\alpha) \approx 0.03 \mathrm{~s}$. 取上升段和下降段的电子束流速度一样, 则可得 $\theta=64^{\circ}$.

\section{2 电子束流的寿命}

引起 U 型爆的电子束流在封闭的磁环中运动, 受到背景等离子体碰撞而衰减, 其寿命应 与整个 $U$ 型爆的持续期差不多. 从图 1 可量得, $U$ 型爆的持续期约为 $6.5 \mathrm{~s}$.

由于库仑碰撞, 在较高密度磁环中的电子束流会很快各向同性化, 碰撞时间为 ${ }^{[5]}$

$$
\tau_{\mathrm{D}}=\frac{m_{\mathrm{e}}^{2} v^{3}}{8 \pi \mathrm{e}^{4} \ln \Lambda N_{\mathrm{e}}} \approx 3.45 \times 10^{-20} \frac{v^{3}}{N_{\mathrm{e}}} .
$$

在日冕情况下, 式中的库仑对数 $\ln \Lambda$ 为 18 . 根据(1)式, 对磁环中电子数密度进行平均, 得 $N_{\mathrm{e}}^{(1)}=3 \times 10^{10} \mathrm{~cm}^{-3}$ (一次谐波), $N_{\mathrm{e}}^{(2)}=7.5 \times 10^{9} \mathrm{~cm}^{-3}$ (二次谐波). 从(4)式计算得 $v^{(1)}$ $=0.59 c, v^{(2)}=0.37 c$. 我们看到, 二次谐波求得的速度与 2.1 节中得到的速度基本上是一 致的. 因此得到结论: 这个 $U$ 型爆是由二次谐波发射的. 这与以前的工作 ${ }^{[1]}$ 的结论是一致 的.

\section{3 环的温度}

冕环中的环顶温度与电子密度和环长由下式表示 ${ }^{[6]}$ :

$$
T_{\mathrm{a}}=6.2 \times 10^{-4}\left(N_{\mathrm{e}} L\right)^{1 / 2},
$$

上下臂的环长 $L=270000 \mathrm{~km}, N_{\mathrm{e}}=3.9 \times 10^{9} \mathrm{~cm}^{-3}$, 得 $T_{\mathrm{a}}=6.3 \times 10^{6} \mathrm{~K}$.

另外, 从图 2 可看出, 不同频率上的时间轮廓基本上类似的, 它们的持续期和衰减时间基 本上相同. 根据文献 [7]中的 (1)式, $T_{\mathrm{f}}=0.65 \times 10^{-4} \tau^{2 / 3} f^{4 / 3}, \tau$ 为衰减时间, $f$ 为频率. 由此 式得环足温度 $T_{f}=2 \times 10^{7} \mathrm{~K}$. 这些温度结果与其它波段上的所得结果也是一致的 ${ }^{[8]}$.

\section{4 环中的磁场强度估计}

束电子被围在环中是由等离子体参数

$$
\beta=3.47 \times 10^{-15} N_{\mathrm{e}} T / B^{2}
$$


小于 1 决定的, 其中 $B$ 为磁场强度. 由上述得到的 $T$ 和 $N_{\mathrm{e}}$ 代入后得 $B^{(1)}$ (一次谐波) $>26.5$ $\times 10^{-4} \mathrm{~T}, B^{(2)}$ (二次谐波) $>9.2 \times 10^{-4} \mathrm{~T} .7260$ 活动区的光球磁场约为 $0.28 \mathrm{~T}$, 用势场推得 在光球上空 $190000 \mathrm{~km}$ 处的环顶磁场 $B \approx 7.0 \times 10^{-4} \mathrm{~T}$. 由此可看出, 一次谐波要求的磁场 太高了, 因此应是二次谐波的磁场较为合理. 这再一次证明这个 U 型爆是由等离子体波的二 次谐波发射的. 不过二次谐波的磁场还是稍微高出势场的场强. 因此在日冕上空应有局部电 流, 这与其它的射电观测结果也是吻合的.

\section{参考文献}

1 Aschwanden M J, Bastian T S, Benz A O et al. Decimetric solar type U bursts: VLA and PHOENIX Observations. Ap J, 1992, $391: 380 \sim 392$

2 Coffey H E ed. Solar-Geophysical Data, part II (582). Boulder: NOAA Environmental Data and Information Service, 1993

3 Newkirk G A. Structure of the solar corona. Ap J, 1961, 133: 983 1013

4 Wild J P. An investigation of the speed of the solar disturbances responsible for type III radio bursts. Australian Journal of Physics, 1959, 12: 369 389

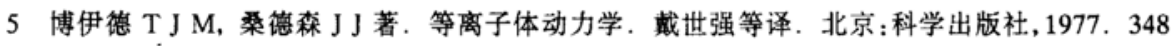

6 Rosner R, Tucker W H, Vaiana G S. Dynamics of the quiescent solar corona. Ap J, 1978, 220: 643 665

7 Alexander J K. Type III radio bursts in the outer corona. Solar Physics, 1969, 8: 388 397

8 Martin S F. Flaring arches I. Solar Physics, 1988, 116: 91 118 